\title{
Open and Closed Layered Nanostructures with Sub-10-nm Periodicity Self-assembled from Hydrophilic [60]Fullerene-based Giant Surfactants
}

Peitao Ding, Xiangfei Yin, Qiyuan Wang, Xiyang Kang, Mei Wu, Ke Zhu, Xiaoliang Wang*, Rong Wang*, and Gi Xue

Key Laboratory of High Performance Polymer Materials and Technology of Ministry of

Education, Department of Polymer Science and Engineering, School of Chemistry and Chemical Engineering, State Key Laboratory of Coordination Chemistry, Nanjing National Laboratory of Nanostructures, Nanjing University, Nanjing 210023, P. R. China

\section{$\underline{\text { Corresponding Authors }}$}

Xiaoliang Wang - Department of Polymer Science and Engineering, Nanjing University, Nanjing 210023, P. R. China. Email: wangxiaoliang@nju.edu.cn

Rong Wang - Department of Polymer Science and Engineering, Nanjing University, Nanjing 210023, P. R. China. Email: wangrong@nju.edu.cn

Number of pages: 15

Number of figures: 10

Number of schemes: 2

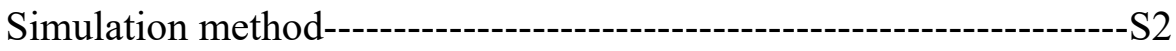

Formation of pupa-like micelle and onion-like micelles----------------S6

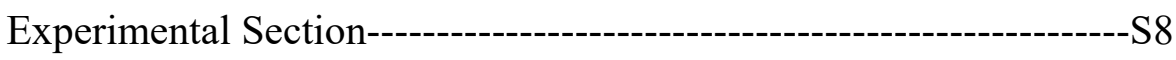




\section{Simulation method}

The dissipative particle dynamics (DPD) method is used to simulate the phase behavior of the giant surfactant. DPD is a coarse-gained particle based mesoscopic simulation technique, which was introduced by Hoogerbrugge and Koelman ${ }^{1}$ in 1992. The DPD approach allows for larger time and length scales than in atomistic molecular dynamics simulations. In a DPD simulation, a coarse-gained DPD particle represents a cluster of atoms and the interactions with each other are short-ranged repulsive forces that contain conservative force $\boldsymbol{F}^{\mathrm{C}}$, random force $\boldsymbol{F}^{\mathrm{R}}$, and dissipative force $\boldsymbol{F}^{\mathrm{D}}$. The time evolution of the simulation system is followed by integrating Newton's equation of motion: ${ }^{2}$

$$
\frac{d \vec{r}_{i}}{d t}=\vec{v}_{i} \quad, \quad \frac{d \vec{v}_{i}}{d t}=\frac{\vec{f}_{i}}{m_{i}}
$$

where $\vec{r}_{i}, \vec{v}_{i}, m_{i}$ and $\vec{f}_{i}$ denote the position, velocity, mass of the $i$ th particle, and the force acting on it, respectively.

The total force acting on particle $i, \vec{f}_{i}$, is the sum of all pairwise interactions as:

$$
\vec{f}_{i}=\sum_{j \neq i}\left(\vec{F}_{i j}^{\mathrm{C}}+\vec{F}_{i j}^{\mathrm{D}}+\vec{F}_{i j}^{\mathrm{R}}\right)
$$

The sum of force acts over all particles within a cutoff radius $r_{\mathrm{C}}$ beyond which the force is neglected. ${ }^{2}$ The conservation force is a soft-repulsive interaction acting along the line of the centers of two particles:

$$
\vec{F}_{i j}^{\mathrm{C}}=a_{i j} \omega\left(r_{i j}\right) \hat{r}_{i j}
$$

where $a_{i j}$ represents the repulsive interaction parameter between particles $i$ and $j$. The weight function $\omega\left(r_{i j}\right)$ provides the range of interaction for DPD particles with a commonly used choice: $\omega\left(r_{i j}\right)=1-r_{i j} / r_{\mathrm{C}}$ for $r_{\mathrm{ij}} \leq r_{\mathrm{C}}$ and $\omega\left(r_{\mathrm{ij}}\right)=0$ for $r_{\mathrm{ij}}>r_{\mathrm{C}}$. 
$\vec{r}_{i j}=\vec{r}_{i}-\vec{r}_{j}, r_{i j}=\left|\vec{r}_{i j}\right|, \hat{r}_{i j}=\vec{r}_{i j} / r_{i j}$. The dissipative force is proportional to the relative velocity, $\vec{v}_{i j}=\vec{v}_{i}-\vec{v}_{j}$ :

$$
\vec{F}_{i j}^{\mathrm{D}}=-\gamma \omega^{2}\left(r_{i j}\right)\left(\hat{r}_{i j} \bullet \vec{v}_{i j}\right) \hat{r}_{i j}
$$

where $\gamma$ is the friction coefficient governing the magnitude of the dissipative force. The random force acts as a heat source to equilibrate the thermal motion of unresolved scales.

$$
\vec{F}_{i j}^{\mathrm{R}}=\sigma \omega\left(r_{i j}\right) \theta_{i j} \hat{r}_{i j}
$$

Where $\sigma$ is the noise amplitude controlling the intensity of the random force, and $\theta_{i j}$ is a randomly fluctuating variable with zero mean and unit variance. The combined effect of the dissipative and random force is that of a thermostat, leading to $\sigma^{2}=2 \gamma k_{\mathrm{B}} T^{2}$

To bind the connected beads of the giant surfactant together, the finitely extensible nonlinear elastic (FENE) potential is added between the consecutive particles. ${ }^{3}$

$$
V_{\mathrm{FENE}}\left(r_{i j}\right)=\left\{\begin{array}{cc}
-\frac{1}{2} k R_{0}{ }^{2} \ln \left[1-\left(\frac{r_{i j}}{R_{0}}\right)^{2}\right] & r_{i j}<R_{0} \\
\infty & r_{i j} \geq R_{0}
\end{array}\right.
$$

where $k$ is FENE spring constant and $R_{0}$ is the equilibrium bond length. We choose $k$ $=30$ and the finite extensibility of the FENE-spring $R_{0}=1.5 r_{\mathrm{c}}$, the choice of $k$ and $R_{0}$ will not affect the qualitative behaviors of the systems studied in this work.

Therefore, we employ the simpler model $\mathrm{H}-\mathrm{T}_{n}$ in our simulations to investigate their self-assembled phase behaviors. The concentration of giant surfactants in the solution $(\varphi)$ is defined as: ${ }^{4}$ 


$$
\varphi=\frac{N_{\mathrm{H}} \times V_{\mathrm{H}}+N_{\mathrm{T}} \times V_{\mathrm{T}}}{N_{\mathrm{H}} \times V_{\mathrm{H}}+N_{\mathrm{T}} \times V_{\mathrm{T}}+N_{\mathrm{S}} \times V_{\mathrm{S}}}
$$

where $N_{\mathrm{H}}, N_{\mathrm{T}}$ and $N_{\mathrm{S}}$ are the number of hydrophilic head beads, hydrophobic tail beads and solvent beads, respectively; $V_{\mathrm{H}}, V_{\mathrm{T}}$ and $V_{\mathrm{S}}$ are the volume of one bead of the hydrophilic head, hydrophobic tail and solvent, respectively. Solvent beads are included explicitly in the simulation; however, they are not shown in the following figures for clarity.

The chosen repulsive interaction parameters are shown in a symmetric matrix:

$$
a_{i j}=\left(\begin{array}{cccc} 
& \mathrm{H} & \mathrm{T} & \mathrm{S} \\
\mathrm{H} & 25 & 50 & a_{\mathrm{HS}} \\
\mathrm{T} & 50 & 25 & 75 \\
\mathrm{~S} & a_{\mathrm{HS}} & 75 & 25
\end{array}\right)
$$

The interaction parameters between particles can be estimated by the relationship between the $a_{i i}$ and Flory-Huggins interaction parameter $\chi_{i j}$ at $\rho=3$ : $a_{i j} \approx a_{i i}+3.497 \chi_{i j}$, where $a_{i i}=25$ for the same type of DPD particles $(i=\mathrm{H}, \mathrm{T}, \mathrm{S})$ to match the compressibility of water. The interaction parameters can be determined from the calculation of dimensionless compatibility. Therefore, the value of $a_{i j} \leq 25$ corresponds to $\chi_{i j} \leq 0$, which indicates that beads $i$ and $j$ are fairly compatible. The value of $a_{i j}$ rises from 25 with increasing incompatibility between $i$ and $j$. Because $\chi N$ can be used to describe the interaction of the block copolymers, the short chains in the DPD method can represent long polymer chains if the $\chi$-parameter increases at the same time. ${ }^{2}$ Thus, the relatively short DPD chains with different interaction parameters can actually also simulate the long polymer chains after applying these parameters. ${ }^{2,5}$

Based on the model polymer and the interaction parameters, we performed the dynamics of total 81000 DPD beads in a cubic box $\left(30^{3}\right)$ under the periodic boundary conditions. The density of all beads in the system is set to 3 . In the present simulations, 
all the hydrophobic polymer tail beads and solvent beads are of the same mass, $m=1$. The interaction cutoff radius for hydrophobic polymer tail beads and solvent beads is set to $r_{\mathrm{C}}=1$ as the unit of length. The size of hydrophilic head $R_{\mathrm{H}}$ is multiples of the hydrophobic polymer tail beads or solvent beads. Generally, the square of the side styrene is $0.7 \mathrm{~nm}^{2}$ and the radius of $\mathrm{AC}_{60}$ hydrophilic head is $1.5 \mathrm{~nm} .{ }^{6}$ Therefore, when we set the diameter of one styrene to 1 , the size of the hydrophilic head should be 1.8 , reasonably. The energy scale is set to $k_{\mathrm{B}} T=1$, where $k_{\mathrm{B}}$ is Boltzmann constant and $T$ is the temperature. The time unit $\tau$ is defined as $\tau=\left(m r_{\mathrm{C}}^{2} / k_{\mathrm{B}} T\right)^{1 / 2}$. The equation of motion was integrated using a modified velocity-Verlet algorithm with $\lambda=$ 0.65. To avoid divergence of the simulation, the DPD step was set as $\Delta t=0.03$, and the amplitude of random noise was set as $\sigma=3.0$. The DPD systems studied are allowed to develop much faster than the molecular dynamics because the DPD approach makes use of soft-repulsive potentials. Therefore, a typical DPD simulation requires only approximately $10^{5}$ steps to equilibrate. ${ }^{2,7}$ In our simulation, each simulation takes at least $1 \times 10^{6}$ steps and the first $3 \times 10^{5}$ steps are for equilibrium. Simulations with different initial random configurations and for various box sizes have also been implemented, which show that the morphology of aggregates at equilibrium is independent of the initial conditions. The different size of the boxes does not influence the formation of aggregations except the number of aggregates in the boxes after equilibrium. ${ }^{7}$ 
Formation of pupa-like micelle and onion-like micelles

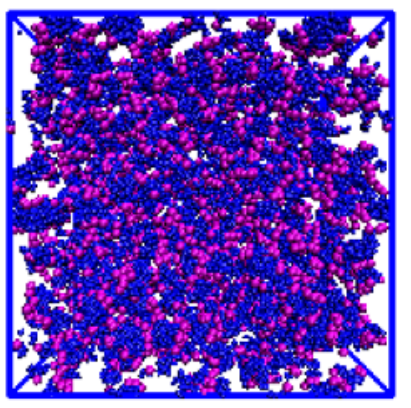

(a)

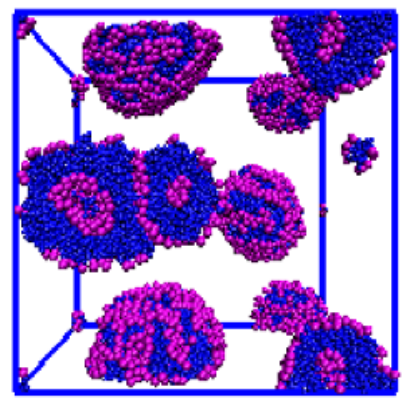

(d)

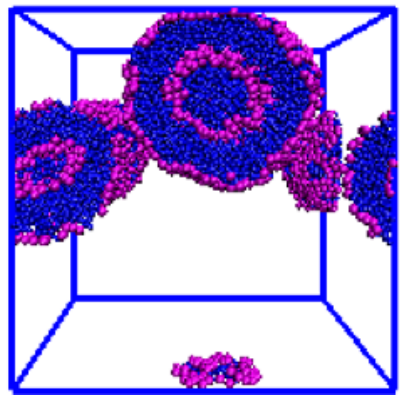

$(\mathrm{g})$

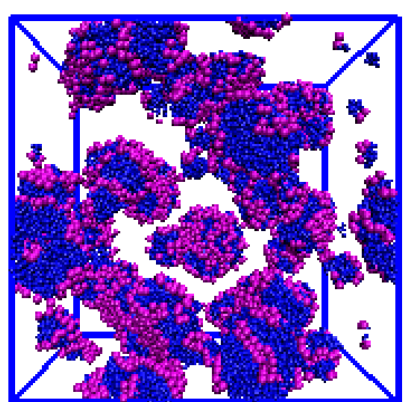

(b)

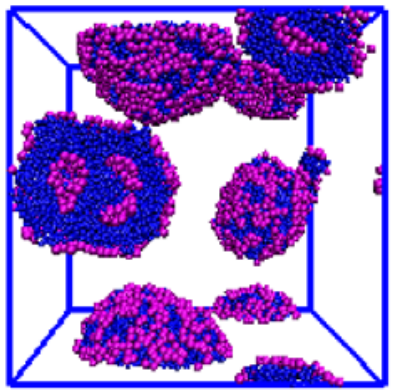

(e)

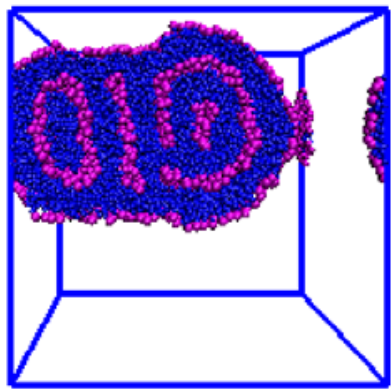

(h)

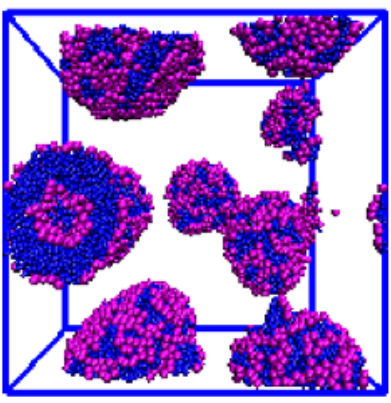

(c)

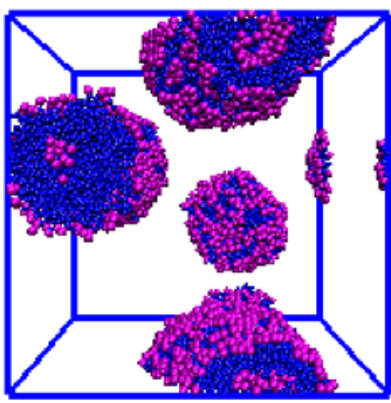

(f)

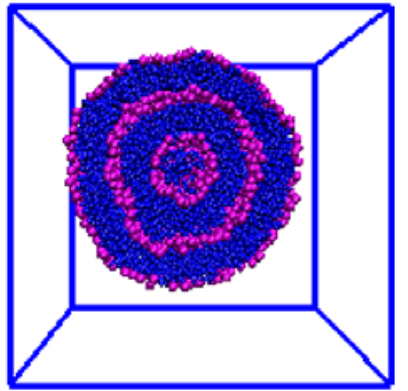

(i)

Figure S1. Snapshots of the formation of the onion-like micelles formed by $\mathrm{HT}_{7}$ with $a \mathrm{HS}=30$ and $\phi=0.175$. (a) $t=30$, (b) $t=300$, (c) $t=1500$, (d) $t=2100$, (e) $t=3000$, (f) $t=4500$, (g) $t=9600$, (h) $t=10500$ and (i) $t=45000$. 


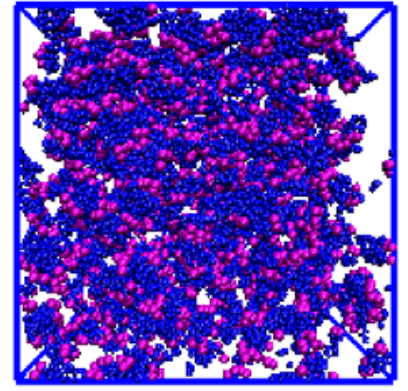

(a)

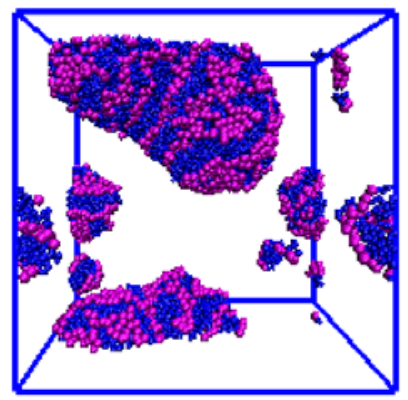

(d)

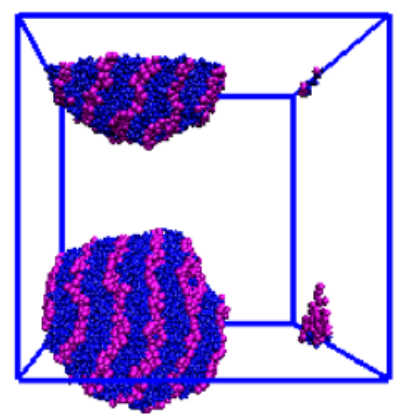

(g)

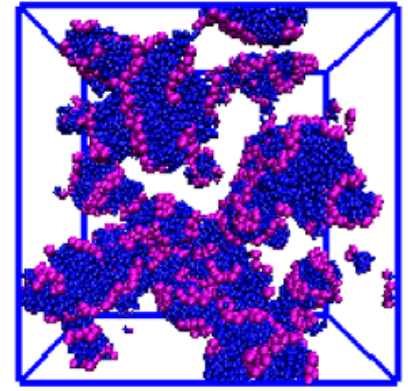

(b)

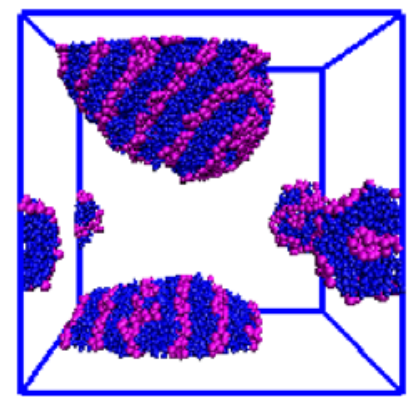

(e)

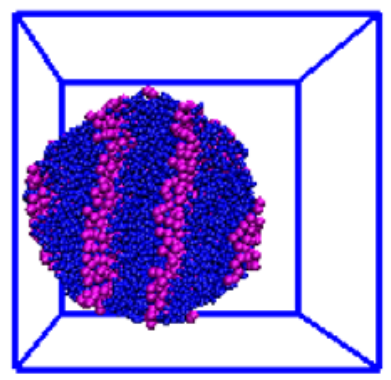

(h)

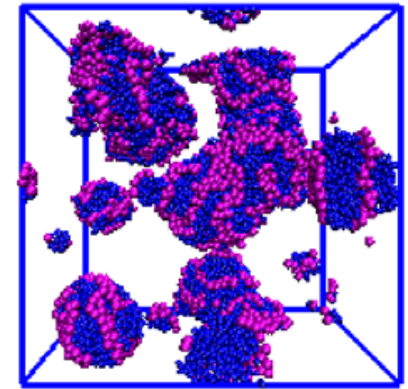

(c)

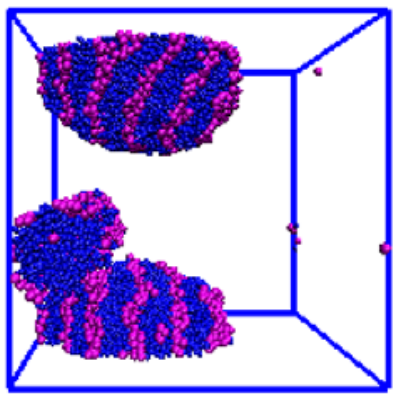

(f)

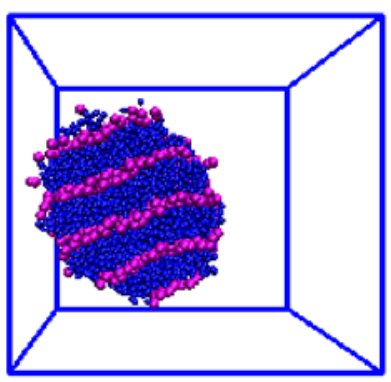

(i)

Figure S2. Snapshots of the formation of the pupa-like micelle formed by $\mathrm{HT}_{7}$ with $a_{\mathrm{HS}}=35$ and $\phi=0.20$. (a) $t=30$, (b) $t=150$, (c) $t=300$, (d) $t=300$, (e) $t=1500$, (f) $t=3000$, (g) $t=6000$, (h) $t=60000$ and (i) $t=60000$. 


\section{Experiment Section}

\section{Characterizations.}

Bright field transmission electron microscope (TEM) images were recorded by a JEM-2100 with an accelerating voltage of $200 \mathrm{kV}$.

NMR spectra were recorded with a Bruker 400M. The concentration of the samples was $10 \mathrm{mg} / \mathrm{mL}$ in $\mathrm{CDCl}_{3}$ or DMSO for ${ }^{1} \mathrm{H} \mathrm{NMR}$ and $30 \mathrm{mg} / \mathrm{mL}$ for ${ }^{13} \mathrm{C} \mathrm{NMR}$.

Matrix-assisted laser desorption/ionization-time of flight (MALDI-TOF) mass spectra were recorded with an ultrafleXtreme TOF/TOF. All spectra were measured in the positive reflection or linear mode. The instrument was calibrated prior to each measurement with external polystyrene standards. For sample preparation, trans-2-(3-(4-t-butyl-phenyl)-2-methyl-2-propenylidene)malononitrile (DCTB) matrix $(20 \mathrm{mg} / \mathrm{mL}$ in THF) and cationizing agent NaTFA $(10 \mathrm{mg} / \mathrm{mL}$ in THF) were mixed with a ratio of $10 / 1(\mathrm{v} / \mathrm{v}) .0 .5 \mu \mathrm{L}$ of the matrix/NaTFA mixture was deposited on microtiter plate wells (MTP 384-well ground steel plate). After the spots were dry, 0.5 $\mu \mathrm{L}$ of each sample $\left(10 \mathrm{mg} / \mathrm{mL}\right.$ in $\left.\mathrm{CHCl}_{3}\right)$ was deposited on top of a matrix/NaTFA spot. After the spots were dried, the target was loaded for data collection.
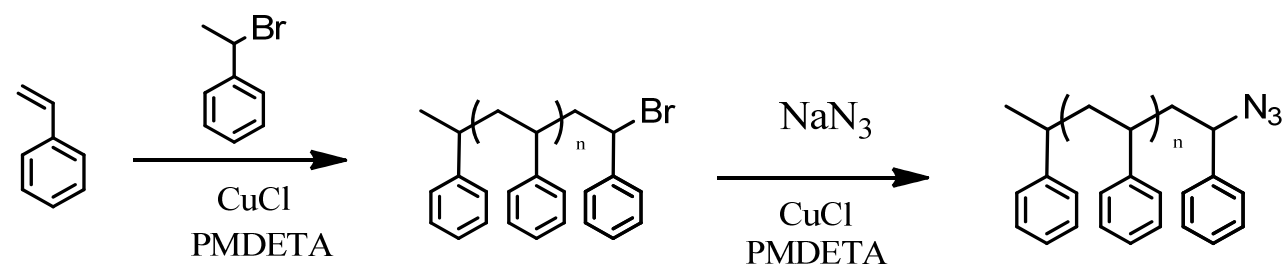

Scheme S1. Synthesis of azido-terminated OS. 


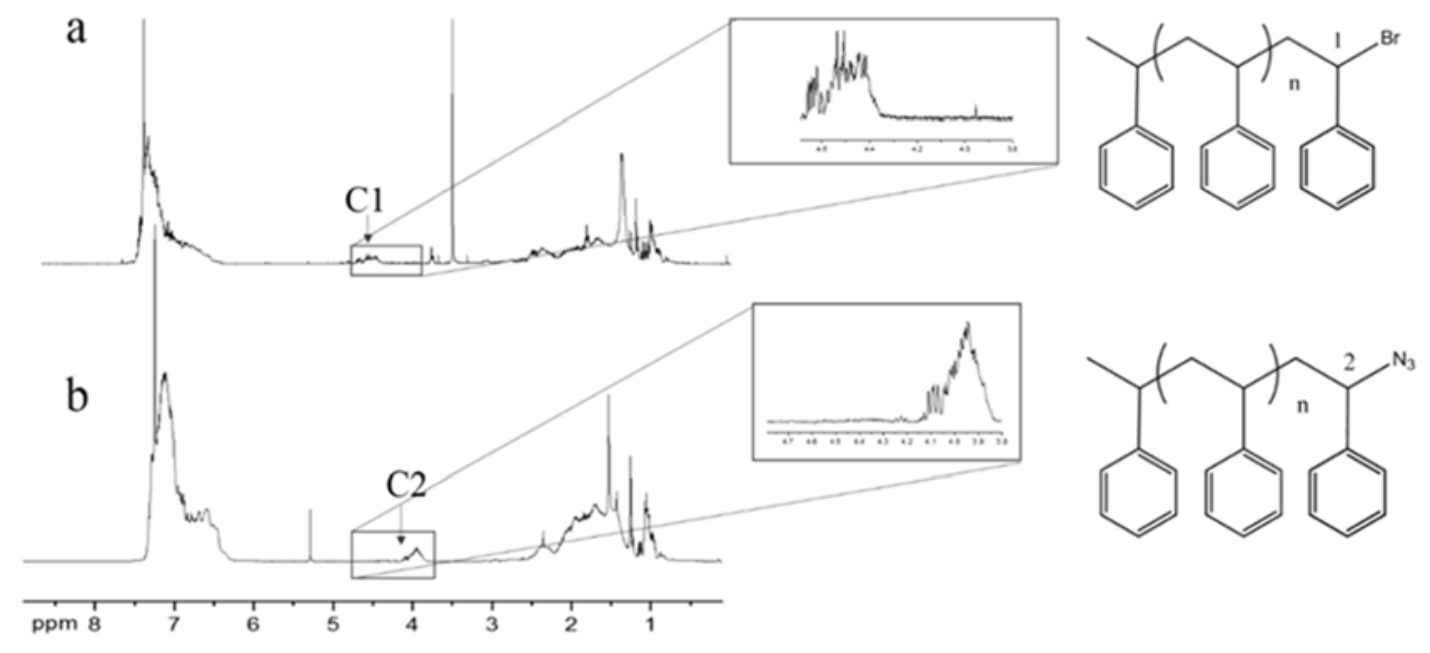

Figure S3. Comparison of ${ }^{1} \mathrm{H}$ NMR spectra of (a) bromo-terminated OS and (b) azido-terminated OS.

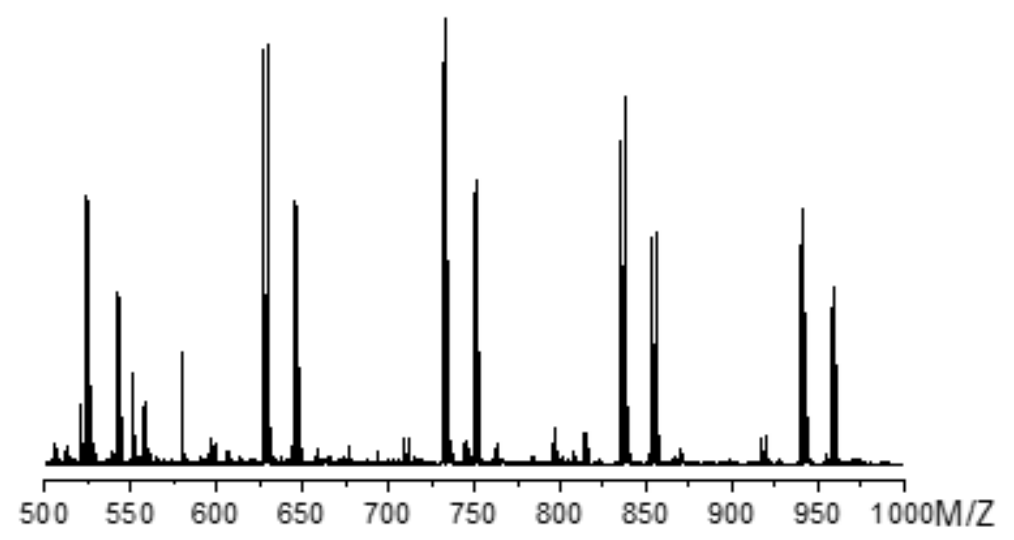

Figure S4. MALDI-TOF mass spectra of azido-terminated OS. 


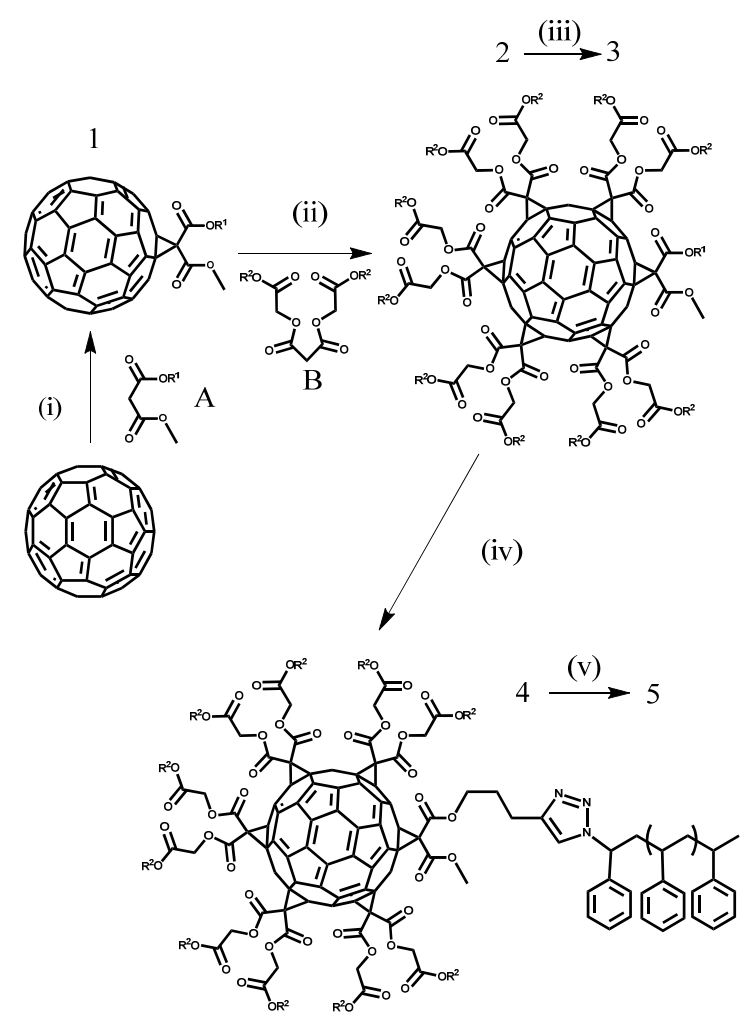

Scheme S2. Synthesis of $\mathrm{AC}_{60}-\mathrm{OS}_{7}$.

$\mathrm{A}: \mathrm{R}^{1}=\left(\mathrm{CH}_{2}\right)_{3} \mathrm{C} \equiv \mathrm{TMS} \quad \mathrm{B}: \mathrm{R}^{2}=\mathrm{tBu}$

1: $\mathrm{R}^{1}=\left(\mathrm{CH}_{2}\right)_{3} \mathrm{C} \equiv \mathrm{TMS}$

2: $\mathrm{R}^{1}=\left(\mathrm{CH}_{2}\right)_{3} \mathrm{C} \equiv \mathrm{TMS} \mathrm{R}{ }^{2}=\mathrm{tBu}$

3: $\mathrm{R}^{1}=\left(\mathrm{CH}_{2}\right)_{3} \mathrm{C} \equiv \mathrm{CH} \mathrm{R} \mathrm{R}^{2}=\mathrm{tBu}$

4: $\mathrm{R}^{2}=\mathrm{tBu}$

5: $\mathrm{R}^{2}=\mathrm{H}$

(i) $\mathrm{C} 60$, toluene, $\mathrm{I}_{2}, \mathrm{DBU}, \mathrm{rt}$

(ii) $\mathrm{ODCB}, \mathrm{I}_{2}, \mathrm{DBU}, \mathrm{rt}$

(iii) TBAF, THF

(iv) $\mathrm{OSn}-\mathrm{N}_{3}$, toluene, $\mathrm{CuBr}$, PMDETA, $\mathrm{rt}$

(v) $\mathrm{CH}_{2} \mathrm{Cl}_{2}, \mathrm{CF}_{3} \mathrm{COOH}, \mathrm{rt}$

The ${ }^{1} \mathrm{H}$ and ${ }^{13} \mathrm{C}$ NMR spectra(Figure S5) and MALDI-TOF mass spectra(Figure S6) were employed to confirm the successful synthesis. 


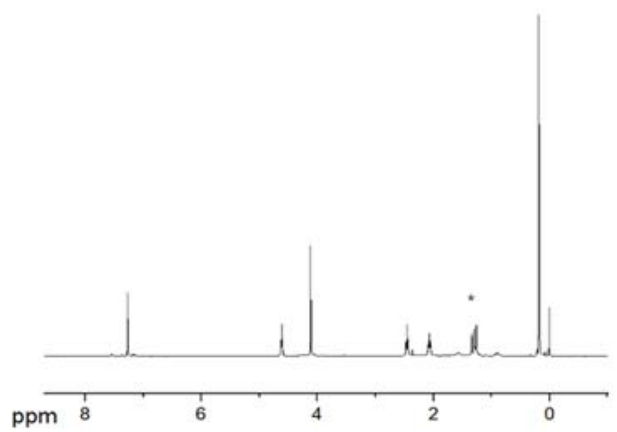

(a)

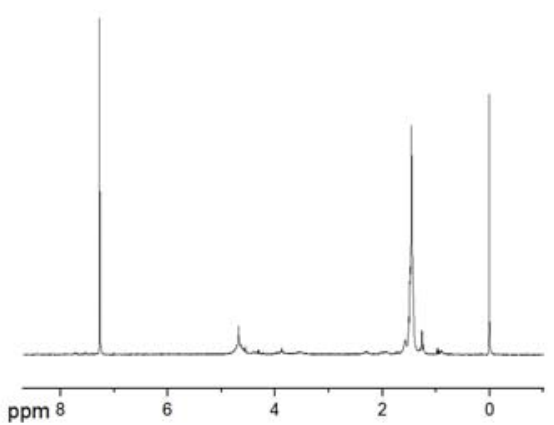

(c)

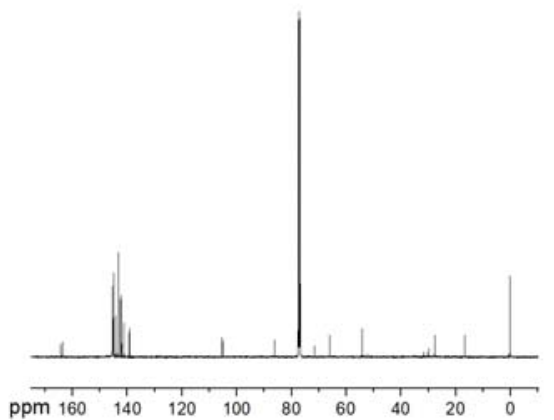

(e)

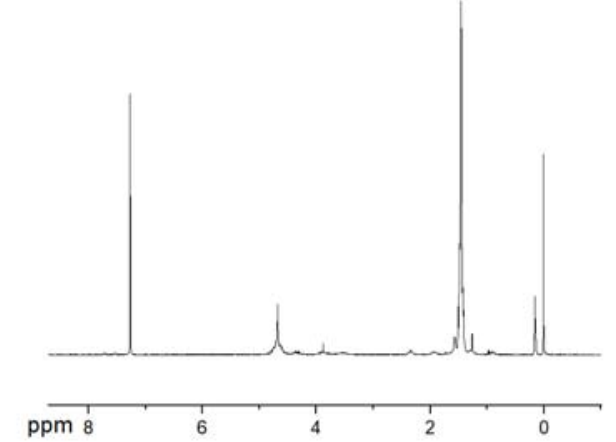

(b)

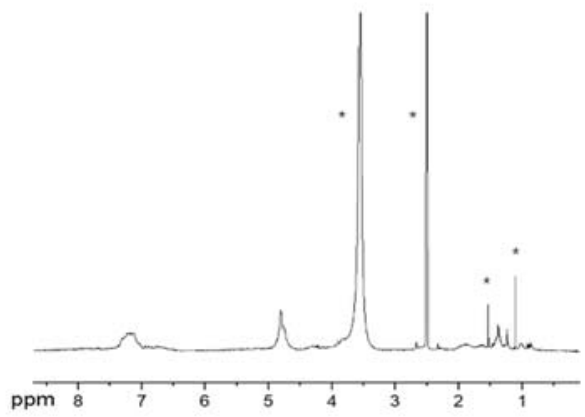

(d)

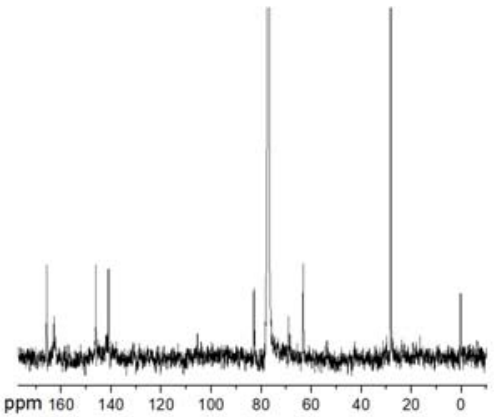

(f) 


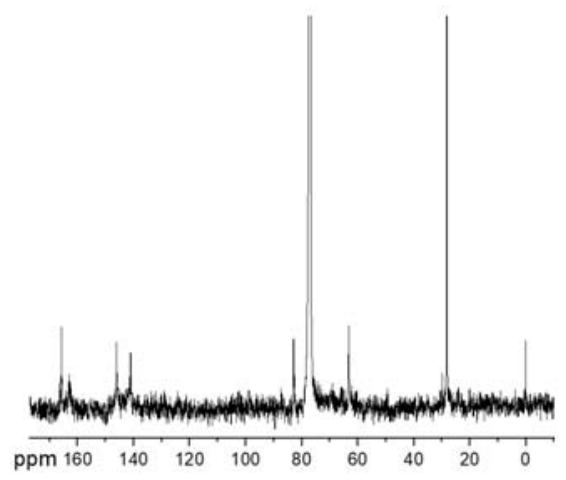

$(\mathrm{g})$

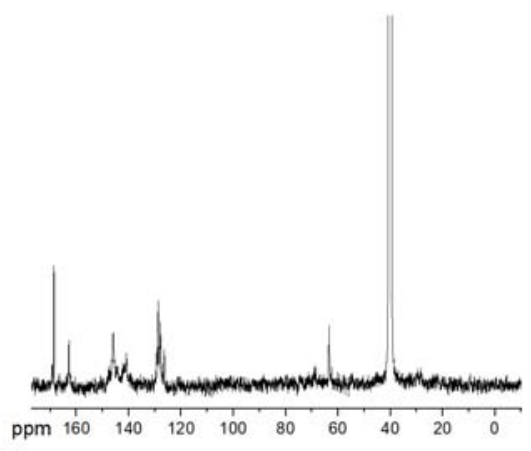

(h)

Figure S5. ${ }^{1} \mathrm{H}$ NMR of (a)1, (b)2, (c) 3, (d) 5 and ${ }^{13} \mathrm{C}$ NMR of (e)1, (f)2, (g) 3, (h) 5 1 5 represent the molecule showed in Scheme S2, and the peaks are listed below:

1: ${ }^{1} \mathrm{H} \mathrm{NMR}\left(\mathrm{CDCl}_{3}, 400 \mathrm{MHz}, \mathrm{ppm}\right.$ ) $) 4.62(\mathrm{t}, 2 \mathrm{H}), 4.12(\mathrm{~s}, 3 \mathrm{H}), 2.46$ (t, 2H), 2.07 (p,

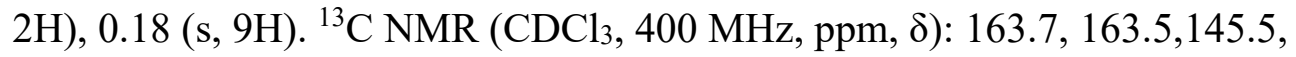
145.4(x2), 145.3(x2), 145.1, 144.9(x2), 144.1(x2), 143.3(x2), 143.2, 142.4, 142.0, 141.2(x2),139.4, 139.1, 105.3, 86.2, 71.7, 66.1, 27.8, 16.0, 0.4.

2: ${ }^{1} \mathrm{H}$ NMR $\left(\mathrm{CDCl}_{3}, 400 \mathrm{MHz}, \mathrm{ppm},\right): 4.68(\mathrm{~s}, 20 \mathrm{H}), 4.36(\mathrm{t}, 2 \mathrm{H}), 3.89$ (s, 3H), $2.36(\mathrm{t}$, 2H), 1.93 (br, 2H), 1.46 (s, 90H), 0.16 (s, 9H). ${ }^{13} \mathrm{C} \mathrm{NMR} \mathrm{(CDCl} 3,400 \mathrm{MHz}, \mathrm{ppm}$,): $165.7,162.9,146.1,141.1,105.6,82.9,69.0,68.4,66.6,63.3,53.8,42.7,28.2,16.6$, 0.34

3: ${ }^{1} \mathrm{H} \mathrm{NMR}\left(\mathrm{CDCl}_{3}, 400 \mathrm{MHz}, \mathrm{ppm}\right.$ ): 4.68 (s, 20H), 4.39 (t, 2H), 3.88 (s, 3H), 2.29 (t, 2H),1.92 (br, 2H), 1.46 (s, 90H). ${ }^{13} \mathrm{C} \mathrm{NMR} \mathrm{(CDCl} 3,400 \mathrm{MHz}, \mathrm{ppm}$,): 165.7, 163.0, $146.1,141.1,82.9,68.9,65.8,63.3,53.8,28.2,0.21$.

5: ${ }^{1} \mathrm{H}$ NMR (DMSO-d6, 400 MHz, ppm,): 6.30-7.30 (br, m, 38H), 5.10-5.30 (br,1H), 4.75 (s, 20H), 4.25 (s, 1H),1.1-2.1 (br, 20H), 0.75-1.00 (br, 3H). ${ }^{13} \mathrm{C}$ NMR (DMSO-d6, $400 \mathrm{MHz}, \mathrm{ppm}):$ 169.3, 166.4,162.9, 145.9, 142.1, 140.8,128.8, 126.4, 68.9, 63.5. 


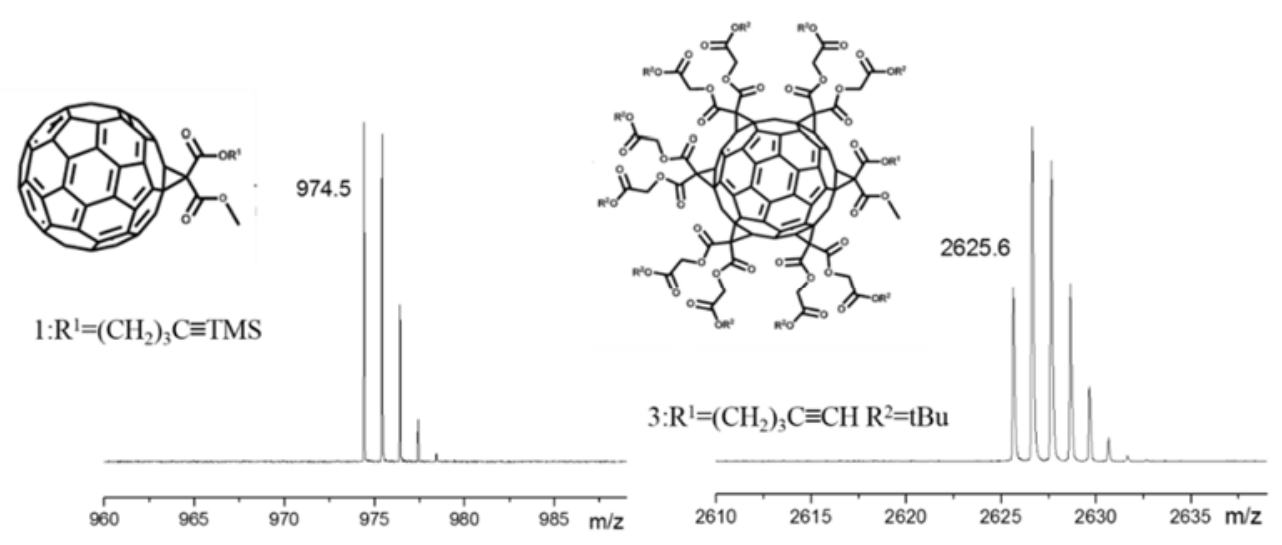

(a)

(b)

Figure S6. MALDI-TOF mass spectra of (a) 1 (Calcd for $\mathrm{C}_{72} \mathrm{H}_{18} \mathrm{O}_{4} \mathrm{Si} \mathrm{M}=974.1$ ) and (b) 3 (Calcd for $\mathrm{C}_{147} \mathrm{H}_{128} \mathrm{O}_{44} \mathrm{Si} \mathrm{M}=2624.9$ ), acquired in positive reflectron mode as molecular radical ions.

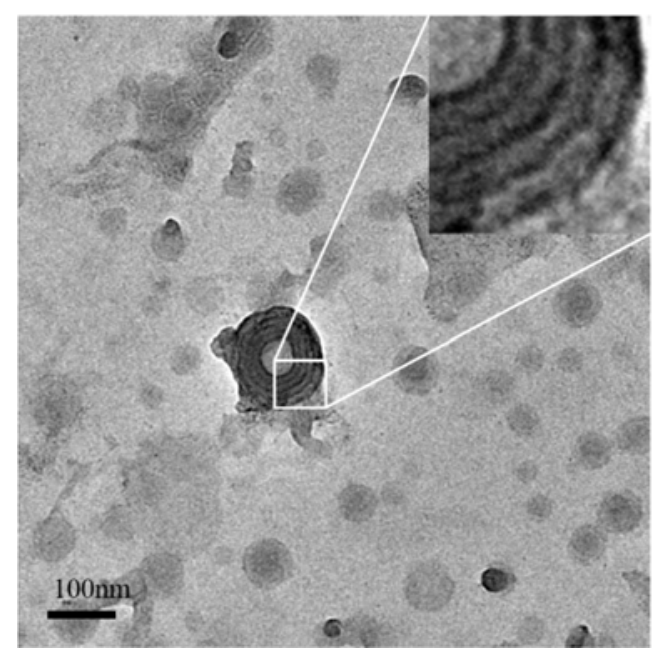

Figure S7. Vesicles with onion-like micelles (scale bar $100 \mathrm{~nm}$ ).

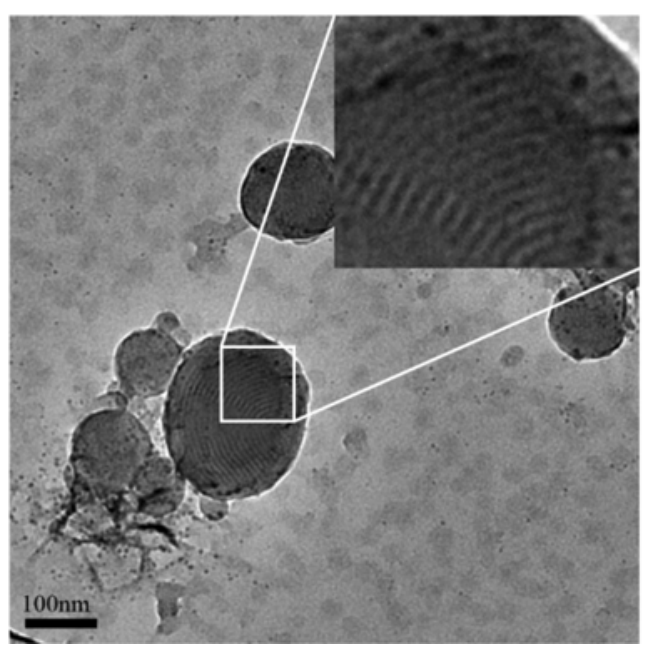

Figure S8. Fat-pupa-like micelles with layers having a big curvature, such that the center of the circle is outside the micelles (scale bar $100 \mathrm{~nm}$ ). 


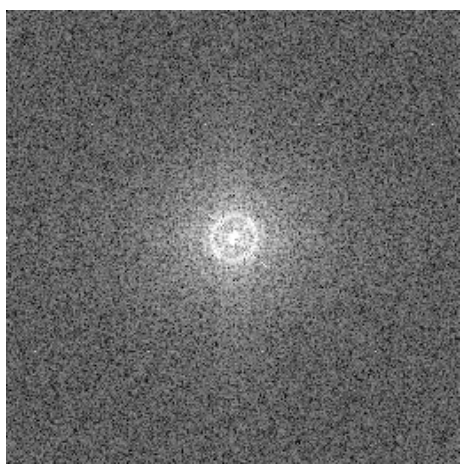

(a)

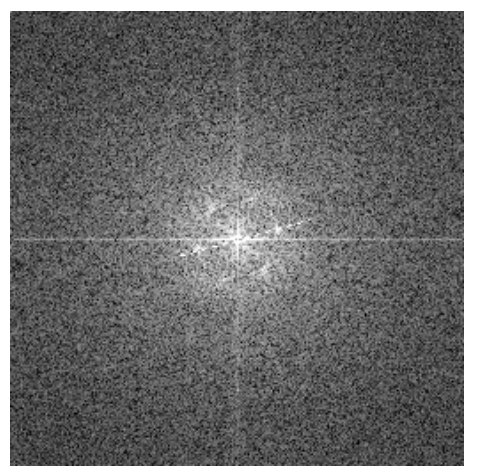

(b)

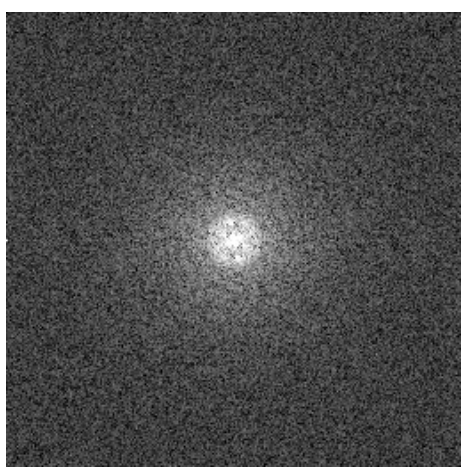

(c)

Figure S9. FFT of before heat: (a) onion-like micelles, (b) fat-pupa-like micelles (c) thin-pupa-like micelles.

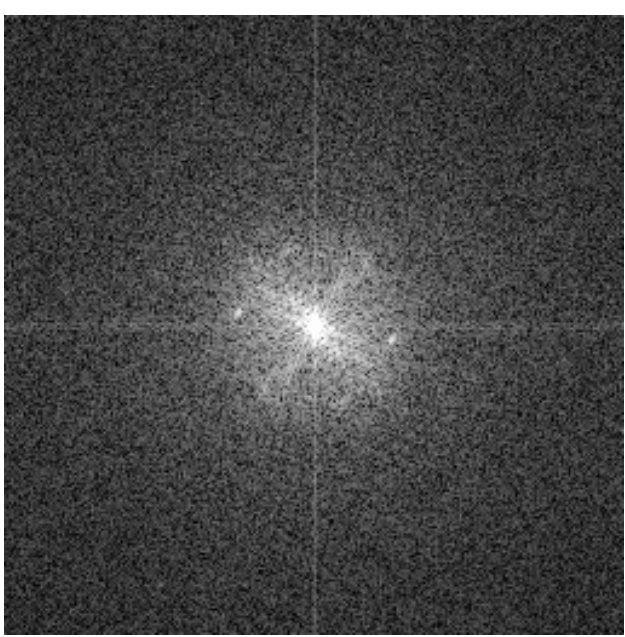

(a)

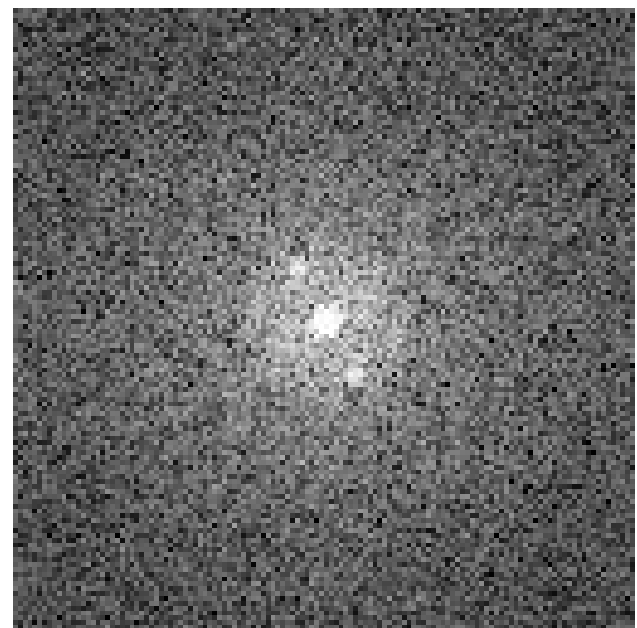

(b)

Figure S10. FFT of after heat: (a) fat-pupa-like micelles, (b) thin-pupa-like micelles.

\section{References}

( 1 ) Hoogerbrugge, P. J.; Koelman, J. M. V. A., Simulating Microscopic Hydrodynamic Phenomena with Dissipative Particle Dynamics. Europhys. Lett. 1992, 19, 155-160. 
(2) Groot, R. D.; Warren, P. B., Dissipative particle dynamics: Bridging the gap between atomistic and mesoscopic simulation. J. Chem. Phys. 1997, 107, 4423-4435.

(3) Kroger, M.; Hess, S., Rheological evidence for a dynamical crossover in polymer melts via nonequilibrium molecular dynamics. Phys. Rev. Lett. 2000, 85, 1128-1131.

(4) Ma, S. Y.; Qi, D. L.; Xiao, M. Y.; Wang, R., Controlling the localization of nanoparticles in assemblies of amphiphilic diblock copolymers. Soft Matter 2014, 10, 9090-9097.

(5) Groot, R. D.; Madden, T. J., Dynamic simulation of diblock copolymer microphase separation. J. Chem. Phys. 1998, 108, 8713-8724.

(6) Yu, X. F.; Zhang, W. B.; Yue, K.; Li, X. P.; Liu, H.; Xin, Y.; Wang, C. L.; Wesdemiotis, C.; Cheng, S. Z. D., Giant Molecular Shape Amphiphiles Based on Polystyrene-Hydrophilic [60]Fullerene Conjugates: Click Synthesis, Solution Self-Assembly, and Phase Behavior. J. Am. Chem. Soc. 2012, 134, 7780-7787.

(7) Chang, H. Y.; Lin, Y. L.; Sheng, Y. J., Multilayered Polymersome Formed by Amphiphilic Asymmetric Macromolecular Brushes. Macromolecules 2012, 45, 4778-4789. 